\title{
MeV-range fast ion losses induced by fishbones on JET
}

\author{
C. Perez von Thun, ${ }^{1}$ A. Perona, ${ }^{2}$ T. Johnson,${ }^{3}$ S. E. Sharapov,${ }^{4}$ \\ M. Reich, ${ }^{1}$ V. G. Kiptily, ${ }^{4}$ M. Cecconello, ${ }^{5}$ A. Salmi, ${ }^{6}$ V. Ya. \\ Goloborod'ko, ${ }^{7}$ S. D. Pinches, ${ }^{4}$ M. García-Muñoz, ${ }^{1}$ D. Darrow ${ }^{8}$ \\ M. Brix ${ }^{4}$ I. Voitsekhovitch,${ }^{4}$ and JET EFDA contributors $\ddagger$ \\ JET-EFDA Culham Science Centre OX14 3DB, Abingdon, UK \\ ${ }^{1}$ Max-Planck-Institut für Plasmaphysik, EURATOM-Association IPP, Garching, \\ D-85748, Germany \\ ${ }^{2}$ Burning Plasma Research Group, Politecnico di Torino, 10129 Torino, Italy \\ ${ }^{3}$ EURATOM-VR Association, Fusion Plasma Physics, EES, KTH, 10044 Stockholm, \\ Sweden \\ ${ }^{4}$ EURATOM/CCFE Fusion Association, Culham Science Centre, Abingdon, OX14 \\ 3DB, UK \\ ${ }^{5}$ EURATOM-VR Association, Department of Physics and Astronomy, Uppsala \\ University, 75120 Uppsala, Sweden \\ ${ }^{6}$ Helsinki University of Technology, Association EURATOM-Tekes, Finland \\ ${ }^{7}$ Association EURATOM/OEAW, Institute for Theoretical Physics, University of \\ Innsbruck, Austria \\ ${ }^{8}$ Princeton Plasma Physics Laboratory, New Jersey, USA \\ E-mail: Christian.Perez.Von.Thun@jet.efda.org
}

\begin{abstract}
Energy and pitch angle resolved measurements of highly energetic (megaelectronvolt range) suprathermal ions ejected from the plasma through interaction with fishbone oscillations are presented. The measurements are obtained with a 2-D scintillator probe diagnostic installed on JET, which is designed to detect lost ions only above a certain energy threshold $\left(E_{\min , \mathrm{D}} \sim 200 \mathrm{keV}\right)$. In the case reported here the lost ions are identified as fast protons which had been accelerated to high energies by ICRF minority heating. The energy of the lost protons $(\sim 0.5-4 \mathrm{MeV})$ is approximately one order of magnitude higher than the energy of the injected beam ions (max. $130 \mathrm{keV}$ ) driving the fishbone. Losses arriving at the probe are enhanced by about a factor 10-20 with respect to MHD-quiescent levels, and are found to increase quadratically with the fishbone amplitude. Using a number of simplifying assumptions, numerical simulations have been performed which combine the HAGIS, MISHKA and SELFO codes (where the distribution function predicted by SELFO has been validated against NPA measurements). The losses are found to originate from orbit stochastic diffusion of trapped protons near the plasma boundary or from counter-passing protons deep in the plasma core which transit under the influence of the fishbone into an unconfined trapped orbit. The simulations show further that the losses are of nonresonant type. The simulated energy and pitch angle distribution of the losses, the
\end{abstract}

$\ddagger$ See the Appendix of F. Romanelli et al., Proceedings of the 22nd IAEA Fusion Energy Conference, Geneva, Switzerland, 2008 
temporal behaviour of the losses during a fishbone cycle and the scaling of the losses with the fishbone amplitude are compared with experiment. The simulation results are mostly in broad agreement with experiment, but some of the predictions could not be reconciled with experiment using this model.

PACS numbers: 52.35.Py, 52.55.Pi, 52.70.Nc, 52.55.Fa, 28.52.Cx

Submitted to: Nucl. Fusion 


\section{Introduction}

To achieve ignition (or high Q-values) in a D-T operated magnetic confinement fusion device and prevent damage to plasma facing wall elements [1], it is necessary that the fast ions generated through auxiliary heating and fusion born $\alpha$ particles remain confined until they transfer their energy to the plasma. Electromagnetic field perturbations generated by the presence of instabilities in the plasma can lead to a premature loss of the fast $\alpha$ 's, either through resonant or non-resonant wave-particle interaction processes $[2,3]$. In many of today's tokamak devices, fishbone oscillations are a commonly observed instability in the sub-Alfvenic range of frequencies. First observed on PDX [4], it is now widely accepted that this mode is destabilised by the presence of a fast (i.e. suprathermal) ion population in the plasma which resonantly interacts with the internal kink mode [5]. Theory predicts [6,7], and experiment confirms ( [2] and references therein), that the resonant ions are in turn subject to enhanced radial transport and loss. In the case of fishbones (or at least for the most commonly observed low frequency type fishbone) the resonant losses tend to have low energy (at most few hundred keV) because at higher energies ions move too fast around the torus and average out the fishbone wave field. For this reason, resonant fishbone losses are not considered to be a source of concern for burning plasmas [8].

However, non-resonant losses of fusion products due to the loss of toroidal symmetry caused by the fishbone were predicted to be important [8]. Indeed, on a number of machines there has been also experimental evidence for fishbone-induced non-resonant losses of highly energetic fusion products. In the PDX tokamak, fishbones were correlated with a reduction in the burnup of $0.8 \mathrm{MeV}{ }^{3} \mathrm{He}$ ions [9]. On DIII-D, both the $1.0 \mathrm{MeV}$ triton and the ${ }^{3} \mathrm{He}$ burnup were found to be anomalously low in the presence of fishbones [10]. On TFTR, the fishbone losses (mainly the $1 \mathrm{MeV}$ triton) could be measured directly with a 2-D imaging scintillation detector [11]. A theoretical explanation has been proposed in [8], but to our knowledge detailed comparisons with experiment have not been made. This paper reports on measurements of $\mathrm{MeV}$ range fishbone-induced fast ion losses obtained on JET with a 2-D scintillation detector [12] and its numerical simulation. The losses have been detected in a variety of discharge scenarios, but for the purpose of this article one particularly well diagnosed discharge was selected for an in-depth analysis. The purpose of this work is twofold. Firstly, the study of this phenomenon is of high interest in itself because of it's possible implications for a fusion reactor. Secondly, to reproduce quantitatively scintillator probe measurements with numerical simulations is not a straightforward task. The relative simplicity of the mode structure of fishbones provides a good opportunity to check the validity of a number of approximations made when trying to reproduce the measurements. The paper is structured as follows. In section 2 a brief description of the setup of the key diagnostics is given. The next two sections of the paper are the main sections. Section 3 presents the diagnostic measurements whereas section 4 reports on the outcome of realistic numerical simulations which were aimed at reproducing the experimental data. 


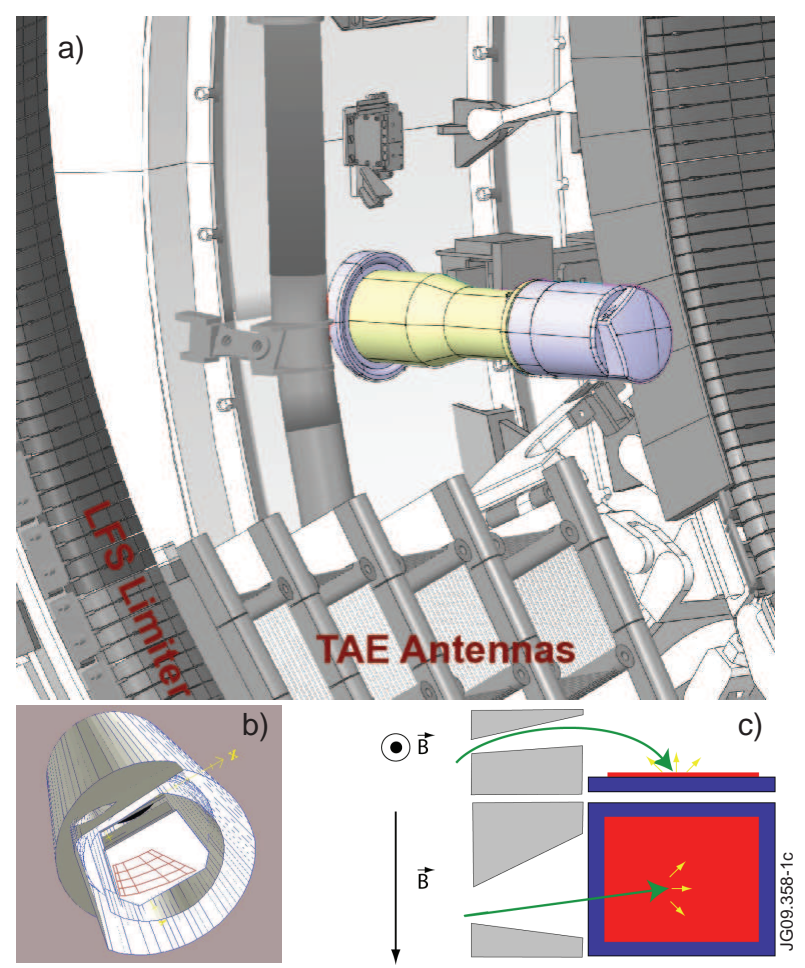

Figure 1. (a) Scintillator probe inside the JET vessel. Also visible to the far left is one of the low field side poloidal limiters, and towards the bottom of the figure a set of 4 TAE excitation antennas. (b) Cross-cut through the probe head showing the collimator and the 2-D scintillator plate. (c) The location at which incoming ions hit the scintillator depends on the gyroradius and the pitchangle of the velocity vector with respect to the local magnetic field.

In the final section a summary of results is provided, the conclusions are drawn and an outlook for future work is given.

\section{Diagnostic Description}

The scintillation detector design is based on the concept of the imaging detector used for the first time on TFTR [13] and more recently in other fusion devices like ASDEX Upgrade [14], CHS [15], LHD [16] and W7-AS [17]. The setup used on JET is shown in figure 1 . The active part of the diagnostic works as a magnetic spectrometer. A 2-D scintillator plate is mounted inside a cylindrical shaft just behind one of the poloidal limiters on the low field side of the torus, approximately 30 degrees below the plasma midplane (standard plasma shapes). A small aperture (collimator) near the end of the shaft is designed in such a way that only escaping ions whose gyroradius and pitch angle lies within a certain range (3-13 $\mathrm{cm}$ and 35-85 degrees, respectively) can enter the probe. The exact position at which those ions hit the scintillator plate and light is emitted yields information on the ions' gyroradius and pitchangle. The ion energy is 
then inferred from

$$
E_{i}=\frac{Z^{2} e^{2} B^{2}}{2 m_{i}} \frac{\rho_{i}^{2}}{\sin ^{2} \vartheta}
$$

(where $Z$ is the ion charge, $m_{i}=A m_{p}$ is the ion mass, $B$ is the magnetic field at the probe 2location, $\rho_{i}$ is the gyroradius, $\vartheta$ is the pitch angle), hence for a given impact point on the scintillator plate the energy of the particles will be dependent on the ratio $Z^{2} / A$ of the ion species. The scintillator light is transmitted through a bundle of $(95 \times 36)$ optical fibers to the diagnostic cubicle. After going through a beam splitter, the light is focussed onto an array of 16 photomultipliers giving high temporal resolution (few $\mathrm{kHz}$ ) and onto a CCD camera yielding high spatial resolution at a somewhat slower rate (50 $\mathrm{ms}$ /frame). The effective time resolution of the diagnostic is limited by the finite decay time for light emission of the scintillator material $(2 \mathrm{~ms})$ and not by the sampling rate of the photomultipliers.

A gold foil ( $1 \mu \mathrm{m}$ thickness) placed between the collimator and the scintillator is used to suppress low energy ions (e.g. protons with $E \lesssim 200 \mathrm{keV}$ ). The remaining faster ions are subject to an energy dependent deceleration due to the foil which is corrected for in our data analysis [18].

It is worth noting that the scintillator probe measurements presented here are all normalised, as an absolute calibration of the diagnostic has not been possible so far. Thus, absolute (quantitative) comparisons between code and experiment are beyond reach at present. Ongoing efforts are underway aiming at the cross-calibration of the scintillator probe against other lost fast ion measurements, notably from a Faraday Cups diagnostic [19] and an Ultra Low Activation Probe [20].

The other main diagnostic used in this work are two neutral particle analysers (NPAs) which yield information on the energy distribution of fast ions confined in the plasma by measuring the energy distribution function of neutral particles escaping from the plasma and the absolute intensity of their fluxes. There is a high and a low energy NPA. The high energy NPA [21] is capable of measuring in the energy range 0.2-4.0 MeV for one species $(\mathrm{H}, \mathrm{D}, \mathrm{T}$ or $3 \mathrm{He}$ ). Its line of sight (figure 2 ) goes vertically through the plasma, for the discharge under investigation at a major radius of 3.10-3.20 $\mathrm{m}$. The low energy NPA [22] measures in the energy range 5-740 keV for $\mathrm{H}, \mathrm{D}$ and $\mathrm{T}$ simultaneously. Its line of sight goes radially through the plasma center.

\section{Experimental Observations}

For the analysis presented here discharge 69100 has been selected because it has excellent diagnostic coverage. It needs to be emphasised that fast ion losses related to fishbone activity are not restricted to this particular case and have been identified in a wide range of discharge scenarios.

During its flat top $(t=21.0-23.4 \mathrm{~s})$ discharge 69100 has an ELMy H-mode with conventional (fully relaxed) $q$-profile and discharge parameters as follows: $B_{0}=2.7$ $\mathrm{T}, I_{p}=1.2 \mathrm{MA}$, edge safety factor $q_{95} \sim 6.5$, normalised beta $\beta_{\mathrm{N}}=2.6$, poloidal beta 


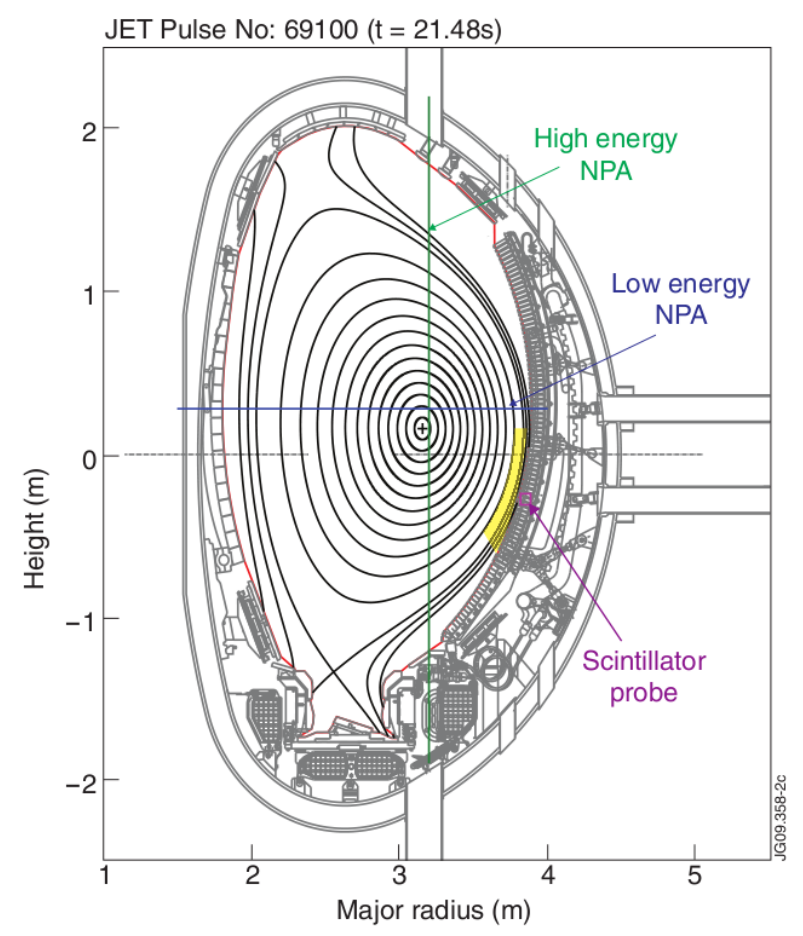

Figure 2. Magnetic equilibrium reconstruction for discharge 69100 . The lines of sight of the low and high energy NPA detectors through the plasma and location of the scintillator probe for lost ions are shown. The yellow area highlights the region near the plasma boundary for which $-60^{\circ} \leq \theta \leq 0^{\circ}$. Lost ions have to cross the separatrix within that area in order to be considered for the simulations results presented in section 4 .

$\beta_{\mathrm{pol}}=1.8$, Greenwald fraction $n_{e} / n_{\mathrm{GW}}=0.77$, triangularity $\delta \sim 0.4$ (the plasma shape is shown in figure 2 ; the plasma centroid is at $R=3.10 \mathrm{~m}$ ). The plasma is composed of 95 percent deuterium and 5 percent hydrogen (inferred from visible spectroscopy measurements at the plasma boundary). The auxiliary heating consists of $15 \mathrm{MW}$ of NBI (max. injection energy $130 \mathrm{keV}$, deuterium) and $6 \mathrm{MW}$ of coupled ICRH (42 $\mathrm{MHz}$, giving a central resonance position of $R=2.82 \mathrm{~m}$ for the hydrogen minority). The heating waveforms are included in figure 3. Both SELFO [24] and PION [25] simulations show that most of the RF heating is coupled to the hydrogen minority population, and that only around 7 percent of the ICRH power (about $0.4 \mathrm{MW}$ ) is damped on the beam deuterons (second harmonic deuterium resonance). Likewise, measurements performed by the neutral particle analysers for hydrogen and deuterium show that for energies well above the maximum NBI injection energy the population of hydrogen exceeds the deuterium population by at least one order of magnitude (figure 3b). By applying the method described in [23] to the NPA data, at the position of maximum RF power deposition along the NPA line of sight a perpendicular tail temperature (mean energy in the perpendicular degrees of freedom) for hydrogen of approximately $240 \mathrm{keV}$ (with 25 percent uncertainty) is inferred (figure 3c).

The MHD activity in this pulse is composed of fishbones and sawteeth in the plasma 

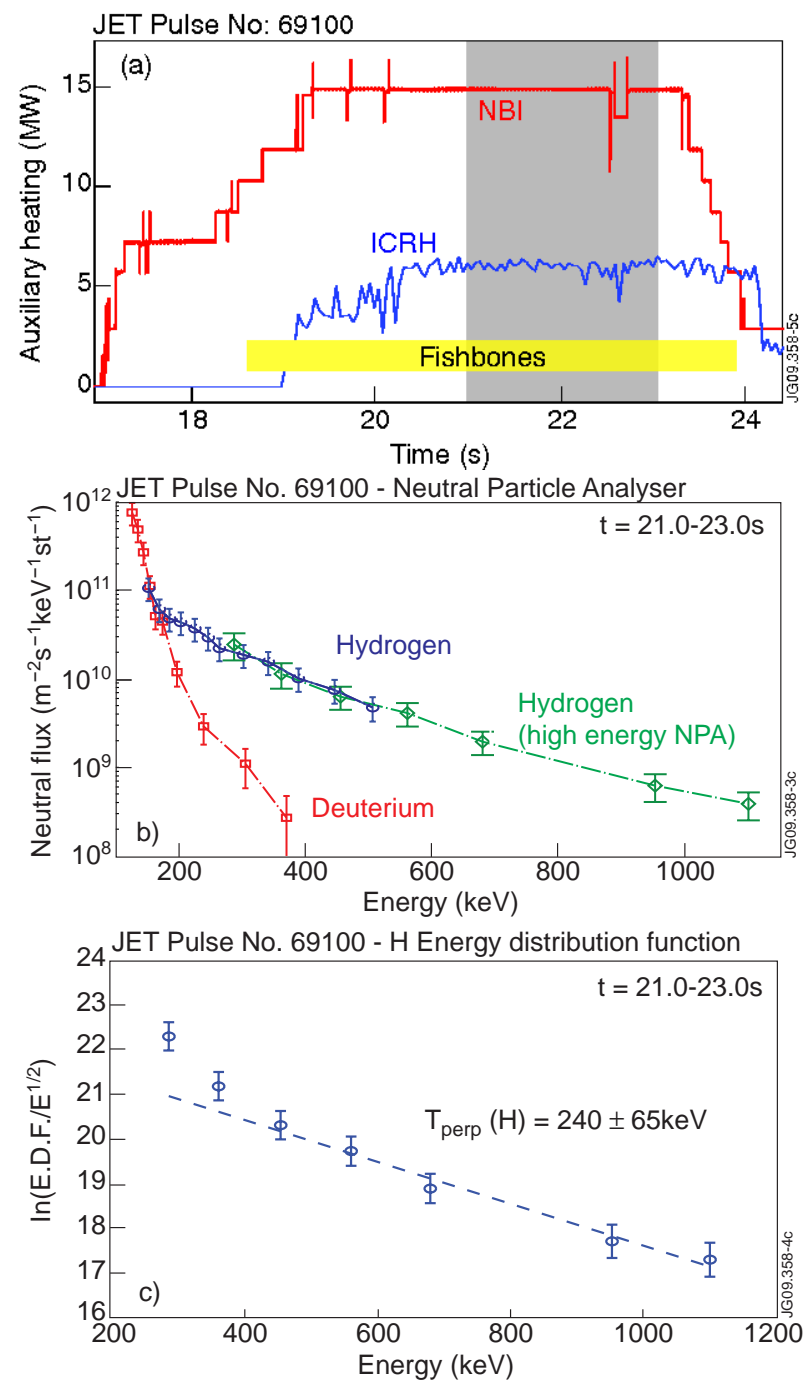

Figure 3. (a) Waveforms of the auxiliary heating in discharge 69100. The grey area marks the time interval used for (b) and (c). (b) Low energy NPA measurements showing the fluxes of hydrogen $(\circ)$ and deuterium $(\square)$ atoms versus energy measured simultaneously along the same line of sight. Also included is the hydrogen flux measured by the high energy NPA $(\diamond)$. (c) Perpendicular tail temperature for hydrogen, inferred from the slope of the energy distribution function measured by the high energy NPA. The energy distribution function is calculated from the measured neutral fluxes using the method described in [23].

core, and small ELMs at the plasma boundary. Other macroscopic activity is not observed with Mirnov coils (maximum available sampling rate: $2 \mathrm{MHz}$ ) or other fluctuation diagnostics (ECE, soft X-ray, reflectometry). Regular fishbone bursts are observable from $t=18.60 \mathrm{~s}$ onwards, i.e. the fishbone activity starts $400 \mathrm{~ms}$ before the ICRF heating is switched on. From $23.4 \mathrm{~s}$ onwards, coinciding with the gradual step down in NBI power at constant ICRH power, the fishbone repetition frequency decreases. The last fishbone is observed at $t=23.92 \mathrm{~s}$, whereas the ICRH power flat top continues until $24.10 \mathrm{~s}$. Also, in a similar discharge run without ICRH heating 
(\#69134, discharge parameters: $B=2.7 \mathrm{~T}, I_{p}=1.2 \mathrm{MA}, q_{95}=6.5, P_{\mathrm{NBI}}=21 \mathrm{MW}$, $\left.\beta_{\mathrm{N}}=2.2, \beta_{\mathrm{pol}}=1.6, n_{e} / n_{\mathrm{GW}}=0.85, \delta \sim 0.4\right)$, the fishbone activity was again present throughout the discharge flat top. The fishbone bursts are correlated with drops in the rate of neutron emission of order $5 \%$ (figure 4). Noting that the neutron production is dominated by the beam contribution (according to TRANSP [26], in this discharge over $95 \%$ of the neutrons originate from beam-target or beam-beam reactions), the above observations clearly demonstrate that the fishbones are driven unstable by the injected beam deuterons, and not the RF accelerated protons, which don't directly contribute in the neutron rate.

In terms of the magnetic fluctuation amplitude $\delta B_{\theta} / B_{0}$ picked up outside the plasma (using magnetic sensors near the outboard midplane), the fishbone amplitude is found to lie in the range $1.0-4.5 \cdot 10^{-4}$. This is a fairly typical value for fishbones on JET [27]. Fourier analysis of soft X-ray emission profiles and of electron cyclotron emission profiles both yield fishbone-induced radial displacements in the range of 3 to $8 \mathrm{~cm}$ for different fishbones (with 20 percent uncertainty) inside the $q=1$ flux surface at the time of maximum fishbone amplitude.

Figure 4 shows a magnetic fluctuation spectrogram and a selection of time traces for a time interval containing four fishbones. On the spectrogram the fishbone oscillations show the characteristic frequency chirp, here from about 14 to $9 \mathrm{kHz}$. The modes visible at twice that frequency are the $m=2 / n=2$ harmonic oscillations (where $m$ and $n$ are the poloidal and toroidal mode numbers, respectively). Also included in figure 4 is one of the photomultiplier signals of the lost ion diagnostic. The occurrence of fishbones is accompanied by a sharp increase in fast ion losses (here factor 10-20 enhancement with respect to background level between fishbones) detected near the plasma edge. These cannot originate from the beam injected ions driving the fishbone. Although beam ion losses are also likely to occur, they are not detectable with this diagnostic due to the presence of the gold foil at the collimator entrance cutting off deuterium ions with less than $200 \mathrm{keV}$. An expanded view for one of the fishbones shows that the ion losses reach their maximum value within the first $2 \mathrm{~ms}$ of the start of the fishbone, slightly delayed with respect to the time of maximum magnetic perturbation (we note that the earlier mentioned finite time constant of the scintillator material limits only the decay rate of light emission, not the rise rate, so the fast rise can still be resolved). After the maximum is reached, the loss signal decreases with a time constant that is comparable to (and thus probably limited by) the finite light decay time of the scintillator material (2 ms). Hence, it is not possible to draw conclusions on the detailed temporal behaviour of the losses in this later phase.

The pitch angle distribution of the detected ions is shown in figure 5. It displays a single peak centered at 58.5 $( \pm 2)$ degrees and with 7.2 degrees broadness (FWHM). From the conservation of the magnetic moment it is found that these ions are on trapped orbits and that for the maximum loss pitch angle the turning points of the orbit, $R_{\text {tip }}$, are in the vicinity (within $6 \mathrm{~cm}$ or less than a gyroradius) of the ICRF resonance position, $R_{\text {res }}(\mathrm{RF})$. Reversely, for trapped ions with turning points located at $R=R_{\text {res }}(\mathrm{RF})$, the 

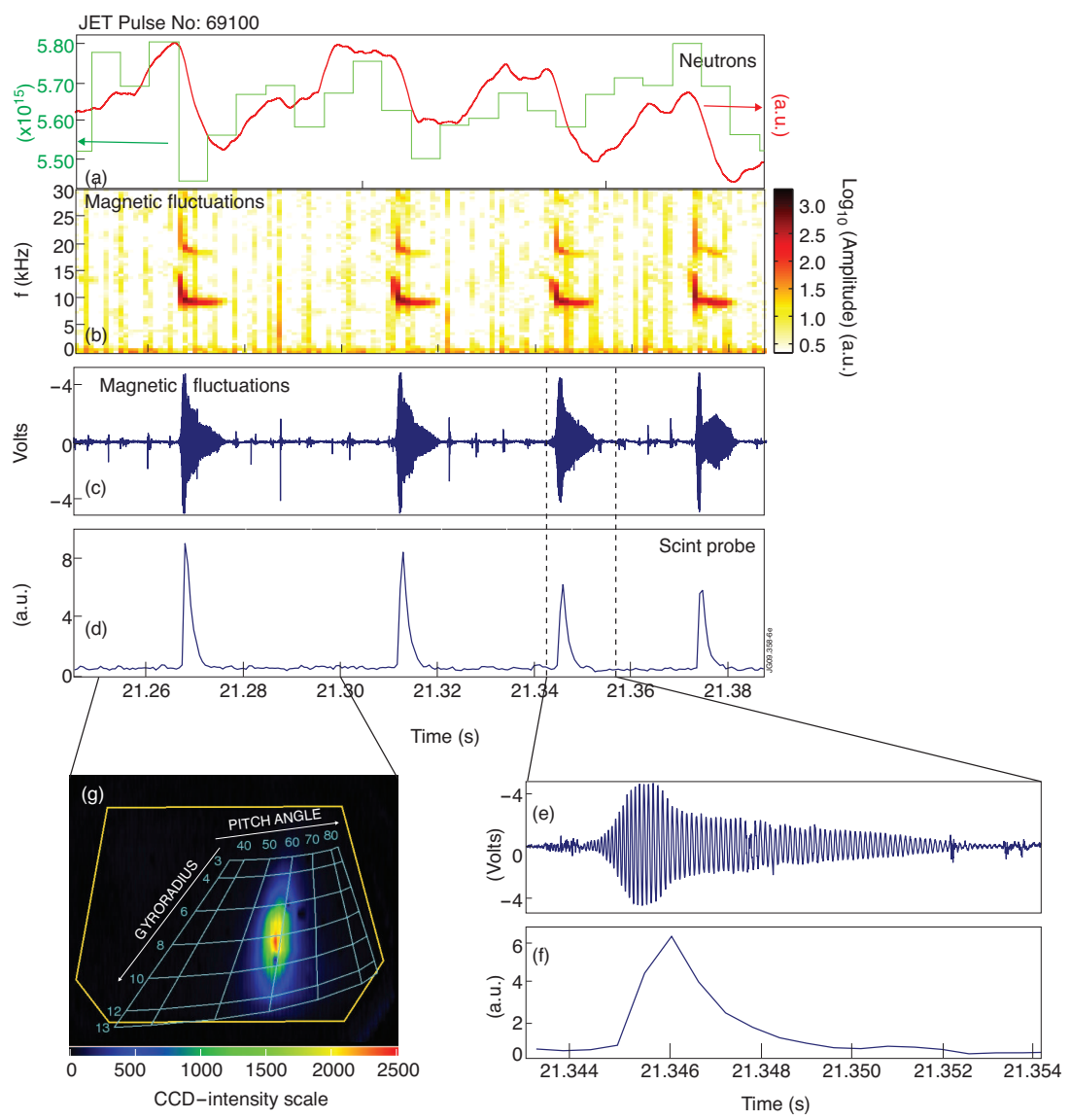

Figure 4. Time interval in discharge 69100 with fishbones: (a): Neutron emission (two traces are shown: are a slow calibrated signal and a faster signal which is uncalibrated), (b): magnetic fluctuation spectrogram showing four fishbones, (c): corresponding magnetic fluctuation time trace (the much smaller bursts also visible on this trace are due to ELMs), (d): scintillator probe signal for one of the photomultipliers, (e) and (f): expanded view of the signals in (c) and (d) for one of the fishbones, (g): CCD camera snapshot of the scintillator emission (where the yellow line marks the contour of the scintillator plate) showing the loss spot, and superimposed to it a grid with the gyroradius (in $\mathrm{cm}$ and not yet corrected for the gold foil) and pitch angle (deg) coordinates.

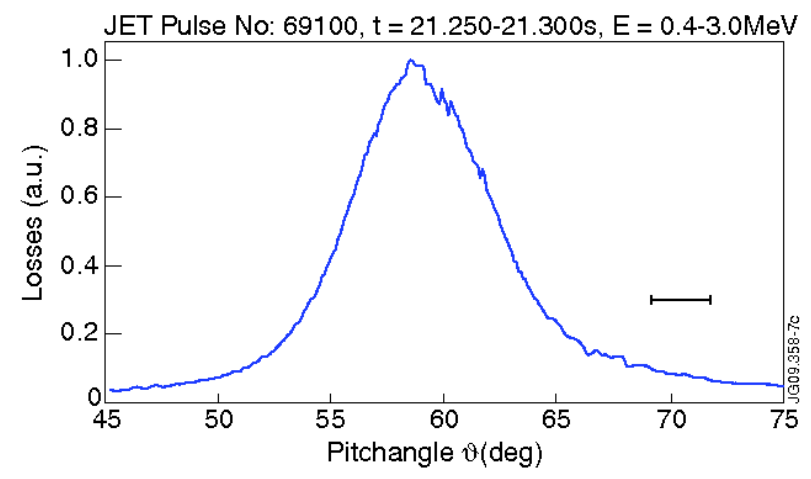

Figure 5. Pitch angle distribution of the fast ion losses for a single fishbone (timeintegrated), measured by the scintillator probe. The error bar gives the finite pitch angle resolution of the diagnostic (which does not vary with the pitch angle). 


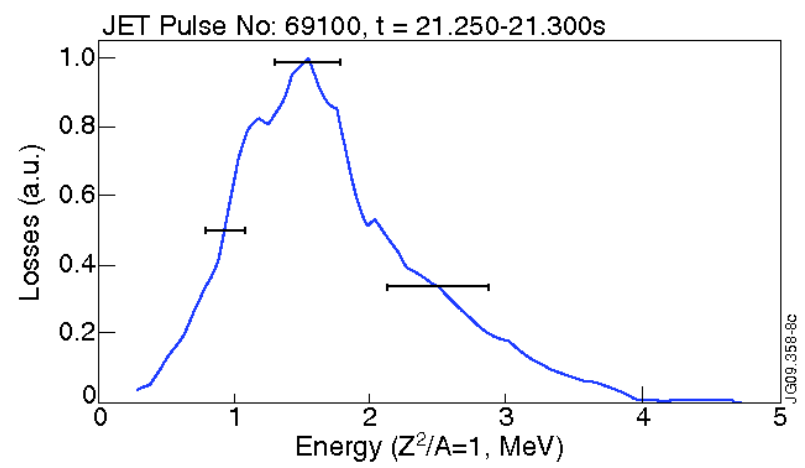

Figure 6. Energy distribution of lost fast ions for a single fishbone (time-integrated), measured by the scintillator probe and assuming $Z^{2} / A=1$. The error bars reflect the finite instrument resolution at various energies (the diagnostic setup is such that the energy resolution decreases with increasing energy).

pitch angle when reaching the probe position would be 59.5 degrees, which agrees with the measured peak maximum within the diagnostic uncertainty. From this observation and given that the fusion cross-section for reactions of $\mathrm{H}$ with the other main species in the plasma is negligibly small (thus ruling out fusion products arising from the protons), it is concluded that the observed losses originate from the ICRH accelerated protons themselves.

The energy distribution of the detected ions is given in figure 6. Having identified the lost ions as protons, $Z^{2} / A=1$ has been used to calculate the energy from the measured ion gyroradius. The losses peak at $1.55 \pm 0.35 \mathrm{MeV}$, with half maximum energies of 1.0 and $2.1 \mathrm{MeV}$, and a high energy tail that reaches in excess of $3 \mathrm{MeV}$. Hence, the lost ions are found to have energies which are at least an order of magnitude higher than the neutral beam injected ions destabilising the fishbones.

\section{Fast Ion Loss Simulations}

Inherent to its design, the scintillator probe diagnostic can only yield information on lost ions arriving at a specific (poloidally and toroidally localised) position inside the torus and hence is unable to give a global picture of the losses. Kinetic simulation codes can be used to obtain more comprehensive fast ion loss predictions (also for ITER), but there is a need to benchmark these codes against experimental data to verify that they do incorporate all the relevant physics. One of the main aims of the scintillator probe diagnostic is to provide this benchmarking possibility. Using a combination of state of the art codes, we test in this section whether the high energy fishbone-induced ion loss data can be correctly reproduced.

To carry out the simulations several steps are involved. First an improved equilibrium reconstruction for discharge $69100(t=22.621 \mathrm{~s})$ is computed by EFIT [28] through additional constraints on the $q$-profile from Faraday rotation angles (polarimetry) and Motional Stark Effect measurements, and on the thermal pressure profile from LIDAR 


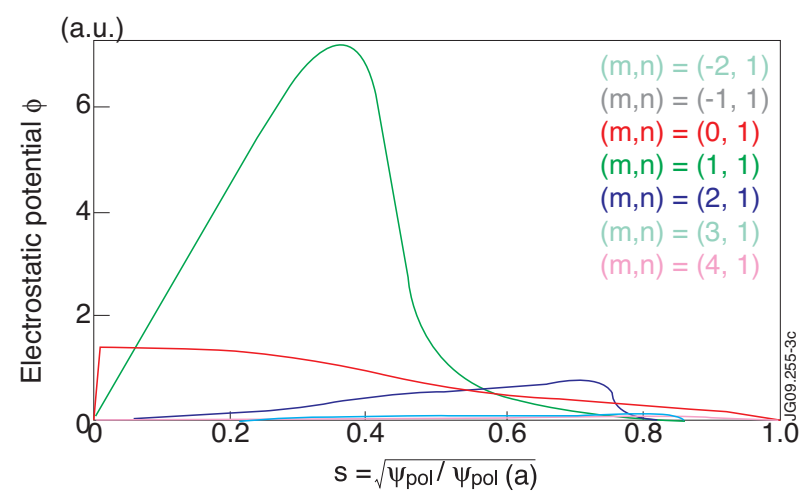

Figure 7. Radial profiles of the electrostatic potential of the $n=1$ internal kink perturbation, computed by MISHKA for pulse $69100(t=22.621 \mathrm{~s})$. The radial coordinate $s$ is the square root of the poloidal magnetic flux. In the legend, $-2 \leq m \leq 4$ denotes the poloidal harmonic.

$\left(n_{e}, T_{e}\right)$ and CXRS $\left(T_{i}\right)$ [29]. The Faraday rotation exploits a stand alone method which has been recently developed to derive profiles of electron density from the far infrared interferometer line integrals [30]. MHD derived $q$-profile information (soft Xray inversion radius for sawteeth, $q_{\min }$ from Alfven Cascades) in a variety of scenarios and comparisons with TRANSP indicate a good agreement with the constrained EFIT $q$-profiles within an error bar of no more than 10-15 per cent [29]. For this particular equilibrium, both the soft X-ray and ECE inversion radii for sawteeth agree well (within $2-3 \mathrm{~cm}$ ) with the reconstructed $q=1$ radius.

The linear ideal incompressible MHD stability code MISHKA-1 [31] is then used to compute the radial eigenfunctions of the $n=1$ internal kink mode (which is a good approximation for the fishbone eigenmode) for this equilibrium (figure 7). The shape of radial eigenfunction is kept fixed over the entire fishbone period (see below), i.e. possible changes to the eigenfunction during the non-linear (finite amplitude) phase [32] are not taken into account. The amplitude and toroidal rotation frequency of the kink perturbation are scaled and chosen to be time-dependent to resemble the typical time evolution of a fishbone. The amplitude is specified analytically through a third order polynomial as follows. For $t \leq t_{\text {sat }}$ :

$$
\frac{A(t)}{A_{\mathrm{sat}}}=\frac{t^{2}}{t_{\mathrm{sat}}^{3}}\left(3 t_{\mathrm{sat}}-2 t\right)
$$

whereas for $t_{\text {sat }}<t \leq t_{\text {period }}$ :

$$
\frac{A(t)}{A_{\text {sat }}}=\left(t_{\text {period }}-t\right)^{2} \frac{\left[3\left(t_{\text {period }}-t_{\text {sat }}\right)-2\left(t_{\text {period }}-t\right)\right]}{\left(t_{\text {period }}-t_{\text {sat }}\right)^{3}}
$$

where $A \equiv \delta \tilde{B}_{r} / B_{0}$ is the radial perturbation amplitude (normalised to the magnetic field on axis), $t_{\text {sat }}$ is the time at which the maximum fishbone amplitude, $A_{\text {sat }}$, is reached, and $t_{\text {period }}$ is the total duration of the fishbone. Figure 8 plots this waveform. From comparison with the experiment, the values chosen for the simulations are $t_{\text {sat }}=1.8$ ms and $t_{\text {period }}=11.0 \mathrm{~ms}$. The value of $A_{\text {sat }}$ has been varied over a wide range. Unless 


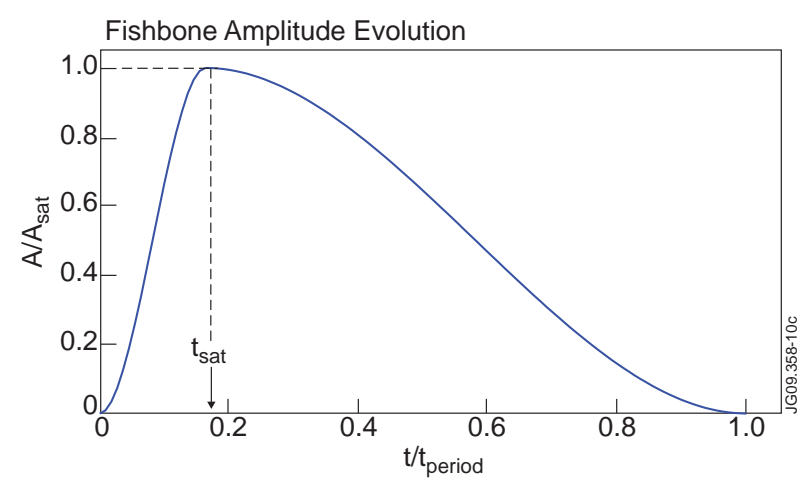

Figure 8. Waveform of the $n=1$ kink amplitude used in HAGIS, including the definitions of the quantities $t_{\mathrm{sat}}, t_{\mathrm{period}}$ and $A_{\mathrm{sat}}$.

otherwise stated, for the following simulations we use $A_{\text {sat }}=1.0 \cdot 10^{-2}$, which, as will be discussed further below, is near the top end of the experimentally observed fishbone amplitudes for discharge 69100. The perturbation frequency is chosen to decrease linearly in time over the fishbone period, from 7 to $2 \mathrm{kHz}$. These values are obtained by subtracting from the measured fishbone fluctuation frequency (figure $4, \sim 14-9 \mathrm{kHz}$ ) the toroidal plasma rotation frequency measured by CXRS, taken at the radial position with maximum perturbation amplitude $(\sim 7 \mathrm{kHz})$. (For completeness, we note that separate tests using the uncorrected frequencies, $\sim 14-9 \mathrm{kHz}$, have shown that the losses are not sensitive to this rotation correction. This is in line with the non-resonant character of the losses, addressed further below). The drift orbit following code HAGIS [33]- release 9.09 - superimposes the 3-D kink mode perturbation computed by MISHKA to the 2-D equilibrium magnetic field configuration.

The initial $\left(t / t_{\text {period }}=0\right)$ fast proton distribution in HAGIS is computed with the SELFO [24] suite of codes, which includes the Monte Carlo Fokker-Planck solver FIDO [34] and the global wave field solver LION [35,36]. It is noted that the Monte Carlo code includes the effect of wide guiding centre orbits and radial transport. The predicted radial distribution for various energy and lambda-parameter $(\Lambda)$ ranges is depicted in figure 9. The lambda parameter is defined as $\Lambda=\mu_{m} B_{0} / E=B_{0}\left(1-\cos ^{2} \vartheta\right) / B$, where $\mu_{m}$ is the magnetic moment and $B_{0}$ is the magnetic field on axis. The advantage of using lambda instead of the pitch angle coordinate is that lambda is an orbit invariant. The radial distribution is double peaked for some energies due to combined inward and outward pinch effects induced by the dipole RF field. It can be also seen that the lambda distribution peaks at values $0.93-0.98$, corresponding to a pitch angle $\vartheta$ of $60-63$ degrees at the probe position.

Figure 10 shows results from a consistency check between the fast proton distribution predicted by SELFO and the experimental measurements obtained by the NPA. Based on the SELFO distribution, the distribution function that a NPA device with vertical line of sight (synthetic diagnostic) would see has been simulated. The same range of energies has been used as for the real NPA diagnostic ( $c f$. figure $3 \mathrm{c}$ ). The simulations 

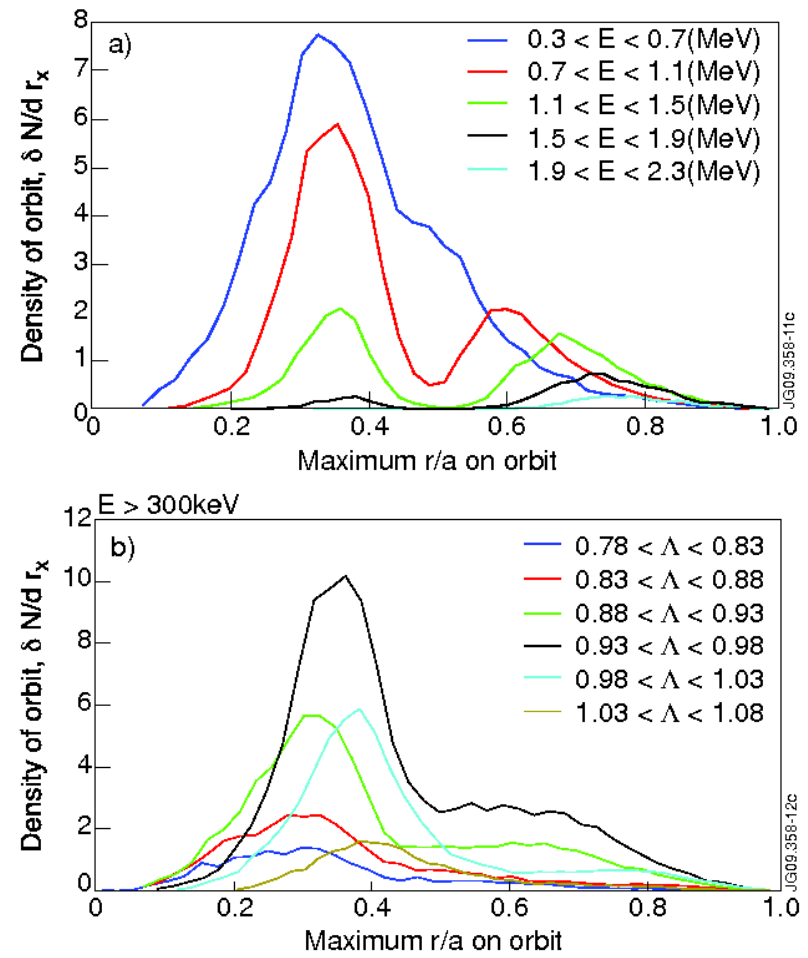

Figure 9. Radial distribution of fast protons predicted by SELFO for discharge 69100 $(t=22.621 \mathrm{~s})$ subdivided into energy (a) and lambda intervals (b). Note that here the representation is done as a function of the maximum (normalised) minor radius of the particle orbit (e.g. for trapped particles, the maximum $r$ reached on the outer leg), not the orbit averaged $r$.

have been carried out for a NPA with line of sight at $R=2.80-2.90 \mathrm{~m}$ (the location of the RF resonance layer) and for a NPA with line of sight at $R=3.10-3.20 \mathrm{~m}$ (the location of the actual NPA diagnostic). Also included for comparison is the energy distribution of the total hydrogen population (for the whole plasma). Figure 10 shows the effective temperatures that have been inferred from the slope of the respective distribution function logarithms. For the line of sight at $R=3.10-3.20 \mathrm{~m}$ a simulated tail temperature of $249 \mathrm{keV}$ is obtained. Within the experimental uncertainty, this is in excellent agreement with the actual measurement (figure 3). Hence, the physics model used in SELFO is able to reproduce the experimental data very well. It can also be seen that near the RF resonance layer a significantly higher tail temperature is predicted $(481 \mathrm{keV})$, which shows that the effective tail temperature varies strongly across the plasma cross section. The average tail temperature of the total proton distribution lies between the two values, at $300 \mathrm{keV}$.

For a first assessment of the losses with HAGIS, a fast $(E>500 \mathrm{keV})$ proton distribution generated by SELFO and composed of 380.000 markers (representing a fast population of $5.5 \cdot 10^{17}$ protons) has been used. (This choice of the number of markers yields enough loss events fulfilling our detection criteria, of order 100, for a first characterisation.) For each of the generated markers HAGIS follows the guiding centre trajectories in 


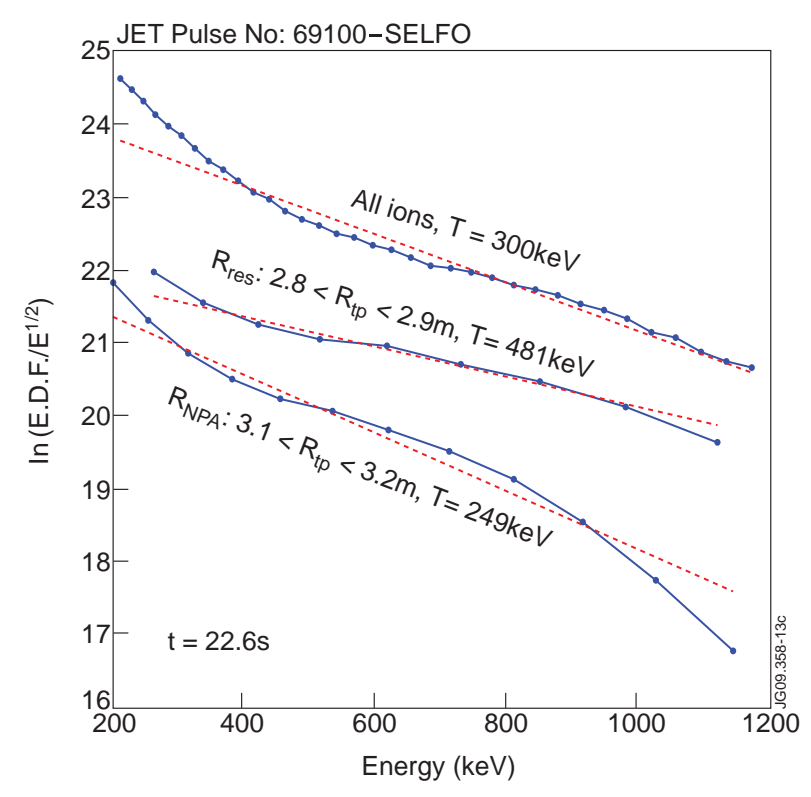

Figure 10. Effective fast proton tail temperatures that a synthetic NPA diagnostic with vertical line of sight would see for the distribution function predicted by SELFO. The temperatures are inferred from the slope of the distribution function logarithm. Three cases are shown: for a line of sight near the RF resonance layer $(2.8-2.9 \mathrm{~m})$, for same line of sight as the actual NPA diagnostic $(3.1-3.2 \mathrm{~m})$, and for the total hydrogen population (whole plasma volume).

the presence of the kink perturbation (as outlined above) during a fishbone period (11 ms). Guiding centres crossing a flux surface near the separatrix $\left(\psi_{\text {pol }}=0.999\right)$ are classified as "lost". This is of course only an approximation, as some of those protons may reenter the plasma without hitting the first wall. Reversely, some protons may hit the first wall at certain locations (notably the LFS limiters) without their guiding centres having crossed the separatrix at any time. The influence of the first wall on the scintillator probe measurements is addressed further below. To account for the poloidal localisation of the scintillator diagnostic, only protons crossing the plasma boundary at a poloidal angle with respect to the plasma midplane of $-60^{\circ} \leq \theta \leq 0^{\circ}$ (cf. yellow area in figure 2) are included. The toroidal localisation of the probe is ignored, i.e. we assume toroidal symmetry of the losses.

When running HAGIS, some of the markers generated by SELFO become lost even in the absence of a magnetic perturbation. These losses occur on a fast timescale ("prompt losses") and have been discarded by running a perturbation-free reference simulation in HAGIS for a time-period of $500 \mu \mathrm{s}$.

The radial origin of the fishbone losses can be quantified by ascribing a quantity $r_{\max }$ to every proton, which we define as the largest minor radius that its unperturbed orbit reaches on the outer midplane. The obtained distribution of the losses against $r_{\max }$ is shown in figure 11. Two groups of losses are observed: (a) from protons close to the plasma boundary $\left(r_{\max } / a>0.9\right)$, and (b) from protons deep in the plasma core (inside or near $q=1$ ). The first type originates from protons on marginally confined trapped 


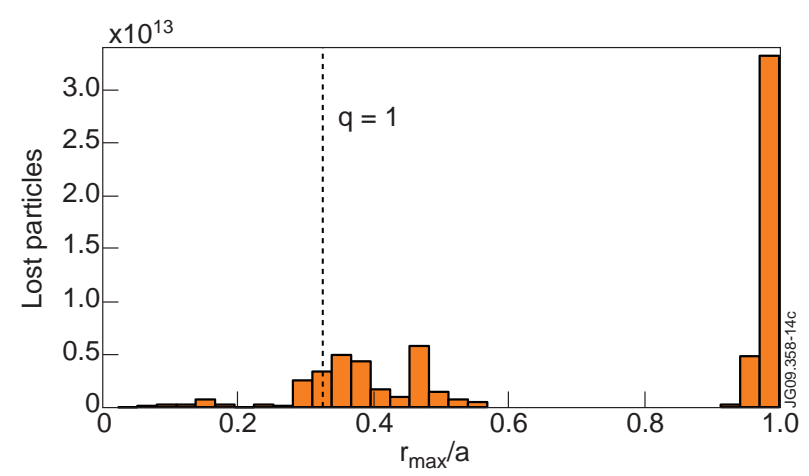

Figure 11. Radial origin of losses predicted by HAGIS for a fishbone with amplitude $A_{\text {sat }}=1.0 \cdot 10^{-2}$ in discharge 69100 . The dashed line denotes the position of the $q=1$ rational surface.
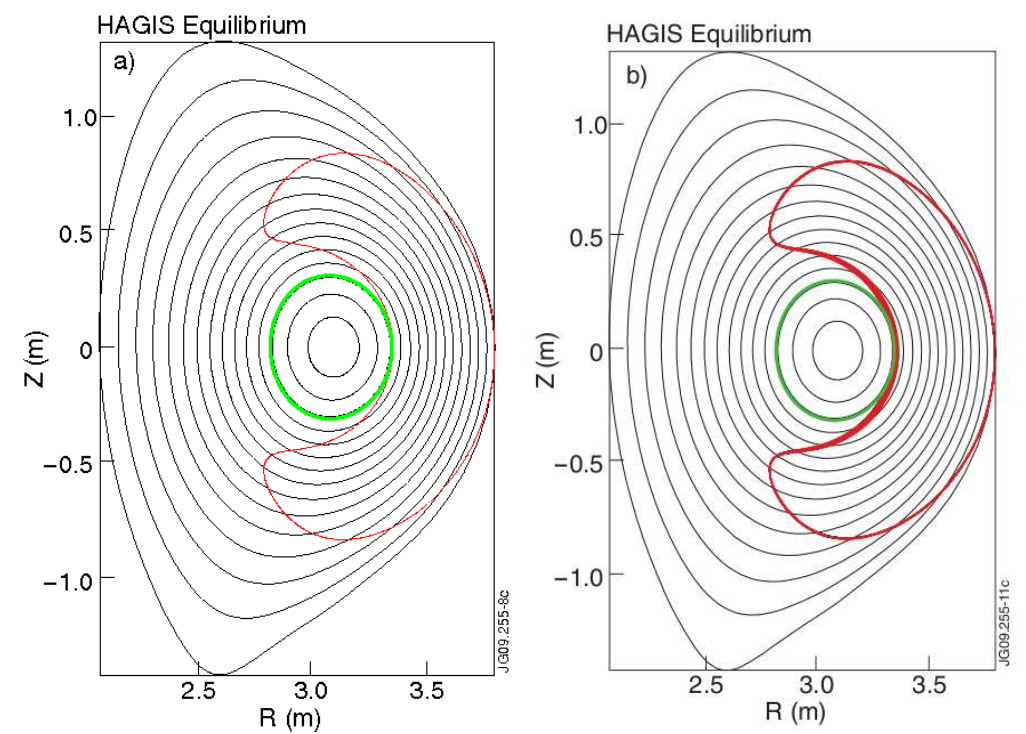

Figure 12. Marginally confined trapped proton $(E=1.36 \mathrm{MeV}, \Lambda=0.89)$ which becomes lost due to the effect of the fishbone perturbation field $\left(A_{\mathrm{sat}}=1.0 \cdot 10^{-2}\right)$ : (a) unperturbed orbit, (b) perturbed orbit. In (b), small deviations from the unperturbed orbit occur, which become visible as a broadening of the red curve after many poloidal bounces. Eventually, the perturbed orbit crosses the separatrix. The green line marks the position of the $q=1$ rational surface.

orbits. One example is shown in figure 12. Even though the radial eigenfunction of the internal kink perturbation has smaller amplitude outside $q=1$, these particles are lost because small deviations of their original orbit suffice to push them across the separatrix. The second type of loss arises from protons with negative pitch which were originally moving on counter-passing orbits inside or near the $q=1$ surface. Due to orbit stochastic diffusion the passing confined ions eventually cross the trapped-passing boundary and become unconfined, as figure 13 shows. For counter-passing ions this will result in an outward radial excursion. If their energy is high enough (which determines the width of the banana orbit), the ions are lost promptly or within a few bounces. One 

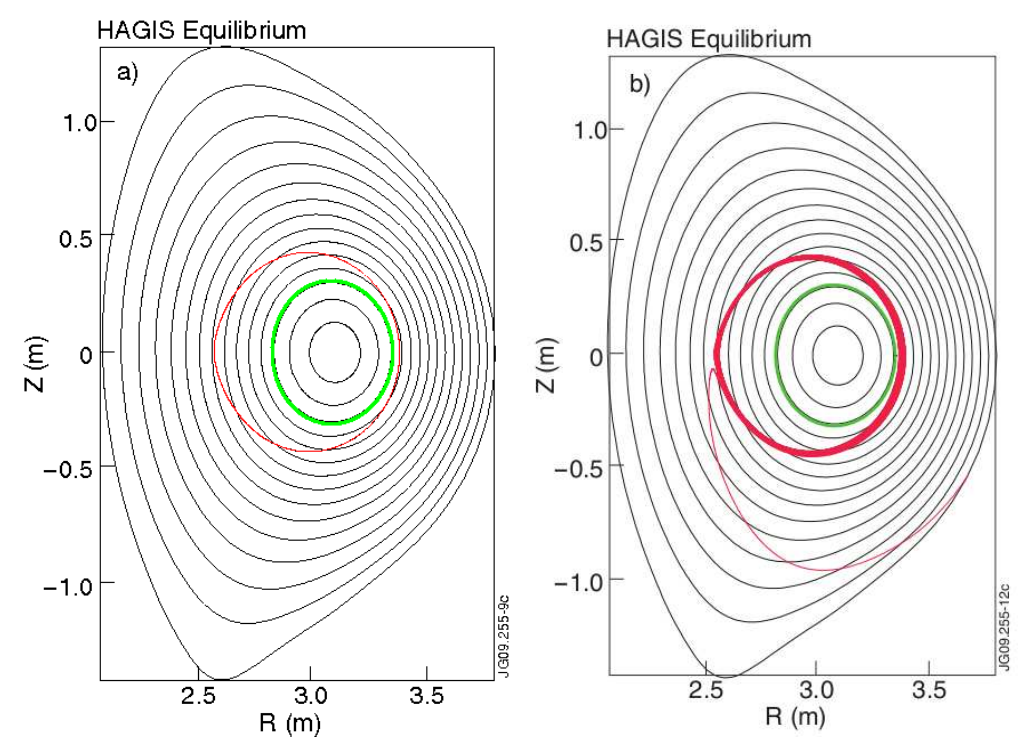

Figure 13. Counter-passing proton $(E=0.94 \mathrm{MeV}, \Lambda=0.81)$ which becomes trapped and lost due to the effect of the fishbone perturbation field $\left(A_{\mathrm{sat}}=1.0 \cdot 10^{-2}\right)$ : (a) unperturbed orbit, (b) perturbed orbit. The green line marks the position of the $q=1$ rational surface.

example is shown in figure 13. Co-moving ions are not lost in this way, because their trapping results in an inward excursion.

Having identified the parts of the proton distribution that contribute to the losses and to further improve the loss statistics, a new set of markers $(E \geq 500 \mathrm{keV})$ was generated with SELFO in which co-moving ions with $r_{\max } / a<0.7$ are omitted, thus maintaining only co-moving ions close to the edge as well as all the counter-passing ions. The new set is composed of 720.000 markers, which, when compared to the first SELFO distribution, is equivalent to using a full fast proton distribution of 9.4 million markers. A HAGIS run with the new distribution requires about 40 peta-Flop (floating-point operations) for one fishbone (roughly 4 days on a set of $100 \mathrm{CPUs}$ with $1.1 \mathrm{GFlop} / \mathrm{s}$ per node).

Figure 14 shows the time evolution of the losses during a fishbone. A comparison with the experimental data shown earlier (figure 4) is limited by the large light emission decay time of the scintillator material, but some features can still be extracted. It can be seen that in both cases there is a fast initial rise of the losses. Moreover, in HAGIS the vast majority of the losses occurs during the first third of the cycle. This finding is at least compatible with the experimental data. One difference found is the time at which the losses peak. In HAGIS, the losses reach their maximum ahead of the maximum perturbation amplitude, whereas in the experiment both maxima are either concurrent or the losses peak is slightly delayed. The reason for this difference has yet to be found. It is unlikely to be linked to the absence in our simulations of a continuous refilling of the fast particle distribution by ICRH, as the characteristic timescales for that process are much longer. Also linked to this, we note that only a small fraction of the overall proton population (sub-percent) is lost, and that simulations run with smaller fishbone 

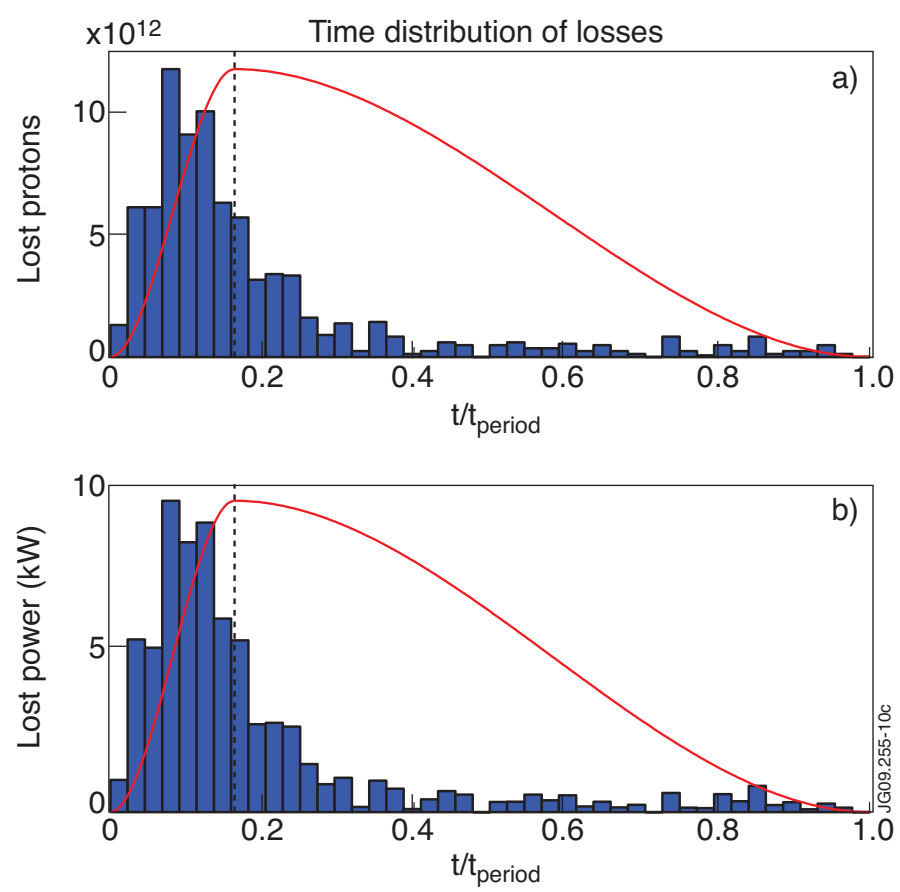

Figure 14. Temporal distribution of proton losses in a fishbone cycle, predicted by HAGIS for a fishbone with amplitude $A_{\text {sat }}=1.0 \cdot 10^{-2}$ (a) Lost number of protons, (b) Lost power. For reference, the magnetic perturbation amplitude is also included in the plot (red curve). The dashed vertical line marks the time at which the maximum perturbation amplitude is reached.

amplitude (leading to smaller losses) still show this time discrepancy, confirming the unimportance of the lack of refilling.

The resonance condition for energetic ions to interact with a wave field is $\Omega_{n p}=$ $n \omega_{\phi}-p \omega_{\theta}-\omega=0$, where $n$ is the toroidal mode number $(=1), p \in \mathbb{Z}, \omega_{\phi}$ is the toroidal ion transit frequency, $\omega_{\theta}$ is the poloidal ion transit frequency (in the case of trapped particles, the poloidal bounce frequency) and $\omega$ is the wave frequency. The above formula is valid for both trapped and passing ions. For each lost ion the proximity to the resonance condition has been examined by inserting for $\omega_{\phi}$ and $\omega_{\theta}$ the original (unperturbed) value (i.e. neglecting variations in $\omega_{\phi}$ and $\omega_{\theta}$ during the fishbone) and maximising the value of $1 / \Omega_{n p}$ by scanning $\omega$ and $p$ (in the range $2 \leq \omega / 2 \pi \leq 7 \mathrm{kHz}$ and $|p| \leq 100$, respectively). Figure 15 plots the number of lost particles against the maximum value of $\log _{10}\left(\left|1 / \Omega_{n p}\right|\right)$ found for each marker. For the vast majority of lost ions $\log _{10}\left(\left|1 / \Omega_{n p}\right|\right)$ remains deeply negative, showing non-resonant character of losses. Also, inspection of the ion energies reveals that for the lost ions $E$ is essentially conserved $(\Delta E / E<0.1 \%$ in nearly all cases), which confirms that there is no energy exchange with the perturbation. It therefore has to be concluded that these fishbone losses are not induced by resonant wave-particle interaction.

Figure 16 shows the energy and the (pitch-angle related) lambda parameter distributions of the lost particles, computed by HAGIS with the new distribution. These are the 


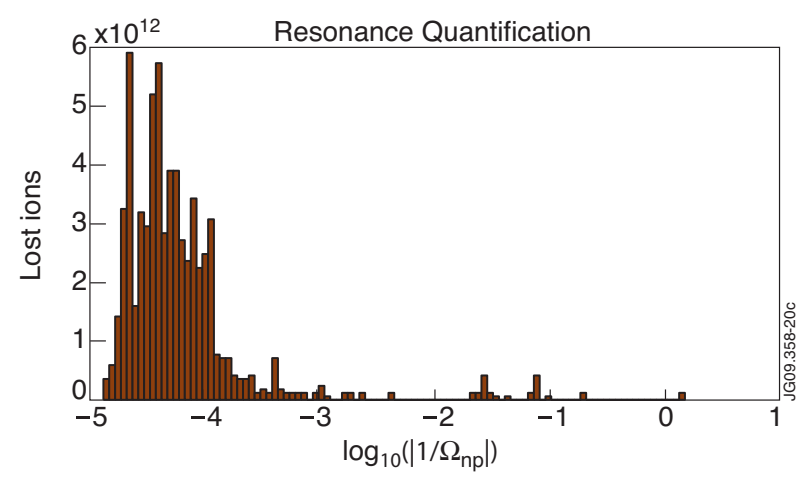

Figure 15. Distribution of losses as a function of the resonance parameter $\log _{10}\left(\left|1 / \Omega_{n p}\right|\right)$ (see text) for a fishbone with amplitude $A_{\text {sat }}=1.0 \cdot 10^{-2}$.
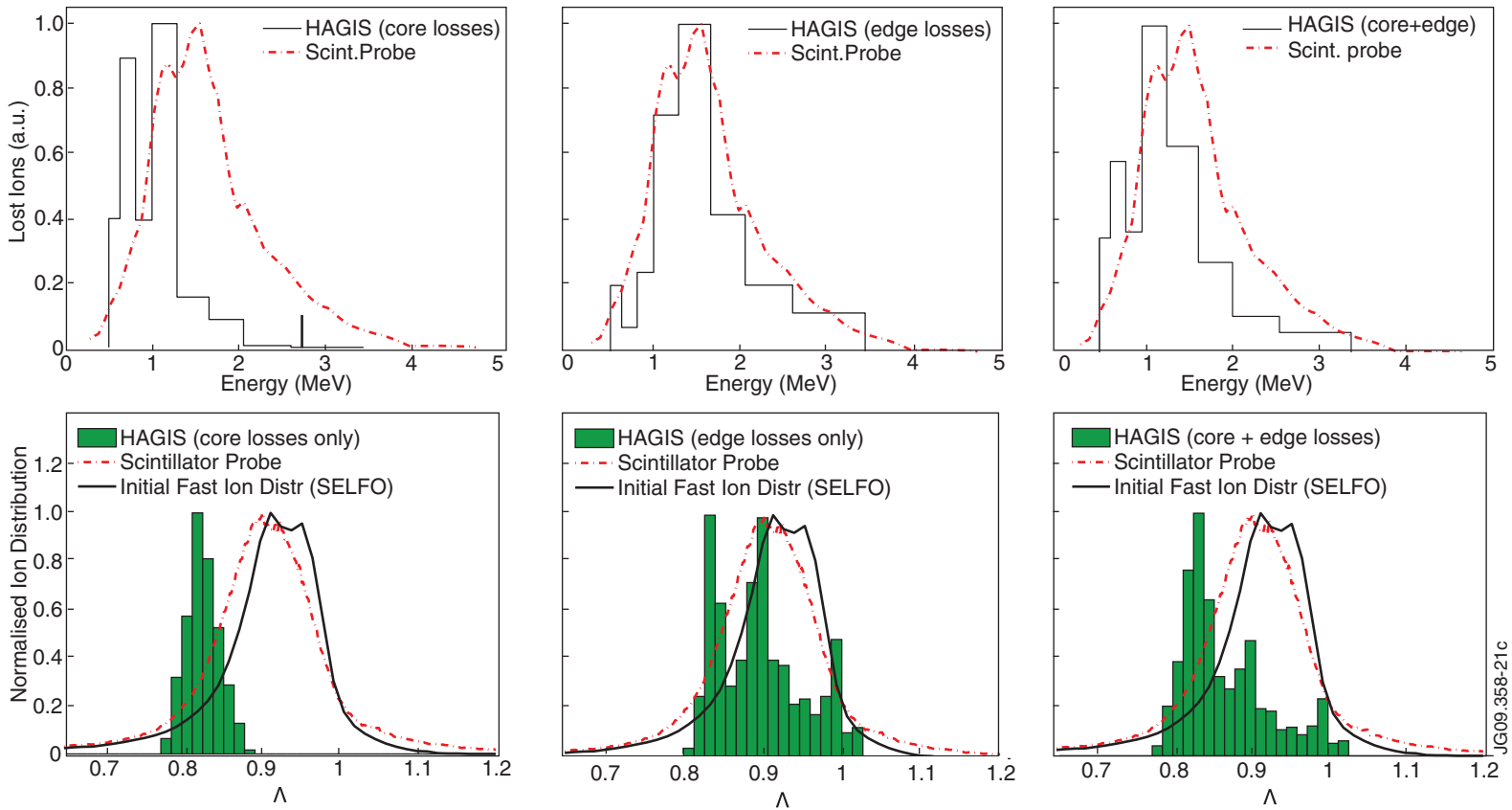

Figure 16. Comparison of measured and simulated lost proton energy distributions (top) and of the measured and simulated lost proton lambda distributions (bottom). In the simulation data only particles crossing the plasma boundary at a poloidal angle with respect to the plasma midplane of $-60^{\circ} \leq \theta \leq 0^{\circ}$ (cf. yellow area in figure 2) are included. (Left): Considering in the simulations only losses from ions initially localised deep in the plasma core (orbits of the type shown in figure 13). (Middle): Considering in the simulations only losses from ions originating from the plasma periphery (orbits of the type shown in figure 12). (Right): Considering all the losses. In the top plots, the width of the energy bins for the simulation data follows the varying instrument resolution of the scintillator diagnostic with energy. In the bottom plots, the initial fast ion lambda distribution computed by SELFO is also included for reference. The simulation data shown uses $A_{\text {sat }}=1.0 \cdot 10^{-2}$. (We note that neither the simulated nor the experimental energy and lambda distributions of losses have shown a strong dependency on the fishbone amplitude.) 
final energy and lambda values of the lost protons, recorded just before crossing the separatrix. The plots also show the measured losses distribution from the scintillator diagnostic, for comparison. In the bottom plots, the lambda dependence of the initial fast ion population predicted by SELFO (here shown with no particle omissions, i.e. with the full co-moving population) is also included. It is instructive to first consider the various loss orbit types separately.

Shown on the left hand side are the results for lost protons originating from deep in the plasma core, $r_{\max } / a<0.8$. This corresponds to loss orbits of the type shown in figure 13. It can be seen that both for the energy and for the lambda distribution the agreement with experiment is poor in this case: The simulated energy distribution peaks at too low energies, and virtually no lost protons are found with energies above $2 \mathrm{MeV}$. In addition, the simulated lambda distribution is shifted with respect to the measured losses, peaking at too low values of lambda.

The middle plots show the results for lost protons originating from the plasma periphery $\left(r_{\max } / a>0.8\right)$. This corresponds to loss orbits of the type shown in figure 12 . For the energy distribution the agreement between simulation and experiment is excellent. Not only do the simulated losses peak at the right energy, but they also follow closely the measured curve over the entire energy range, with a steep increase at around $1 \mathrm{MeV}$ and a high energy tail reaching up to $3.5 \mathrm{MeV}$. In the lambda distribution, the lost markers now display the right range of lambda values, but rather than exhibiting a single peak the distribution looks fairly random. The reason for this is unclear. It is particularly surprising given that initially (before applying the fishbone perturbation) the fast ion distribution had already closely resembled the lambda distribution measured by the scintillator probe. Furthermore, it turns out that the lambda distribution is barely modified by the fishbone: The simulations show that for any single marker the final value of $\Lambda$ barely deviates from the initial one $(\Delta \Lambda / \Lambda$ never exceeds $0.3 \%)$, as predicted by theory for waves with frequencies well below the ion cyclotron frequency.

Against initial expectations, combining all the losses predicted by HAGIS (from core and edge regions) does not lead to the best data fit (figure 16, right). In fact, the agreement is poorer than in the middle plots, which suggests that the contribution of the core-type losses relative to the edge-type losses is significantly overestimated by the simulations. Another possibility would be that the core-type losses take place but cannot reach the probe location due shadowing effects of nearby first wall components. In this context two wall elements are of particular concern: a neighbouring low field side poloidal limiter and an array of TAE excitation antennas (both are visible in figure 1). To check their possible impact on the measurements, orbit following calculations with a 3-D model of the JET wall were carried out in which particles where started at the scintillator probe entrance slit and traced backwards in time. Two Monte Carlo codes were used independently: guiding centre code ASCOT [37] (here the particles were started one gyroradius away from the slit) and a full orbit following code developed at Innsbruck [38]. The energy and pitch values of the launched test ions were scanned in order to cover the full range measurable by the diagnostic (e.g. ASCOT launched 


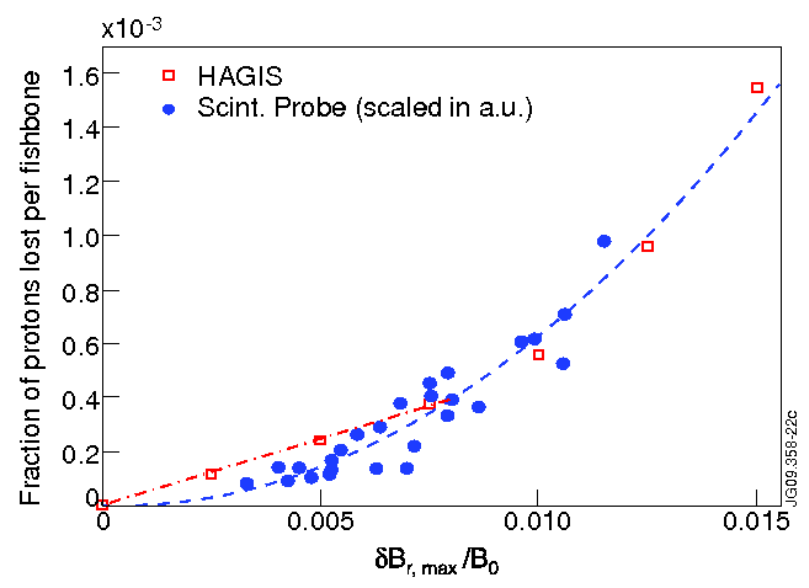

Figure 17. Measured and simulated losses dependence on the fishbone amplitude. For the experimental data, the fishbone amplitude parameter $\delta B_{r, \max } / B_{0}$ has been inferred by matching flux surface displacements on magnetic field line Poincare plots with ECE temperature profile displacements measured inside $q=1$.

protons with $100 \mathrm{keV}$ to $5 \mathrm{MeV}$ and positive pitch from 0 to 1 ). Because here we are interested only in orbits of the type shown in figure 13, it is sufficient to trace the ions backwards in time over a single bounce. Both codes show that for this particular magnetic field configuration neither the limiter nor the antenna array have a shadowing effect on the measurements. The TAE antenna does not capture any of the ions because the B-field pitch is too low, whereas the high plasma triangularity moves the ions away from the limiter. As shadowing effects are unimportant, our results indicate that the simulations greatly overestimate the core-type losses relative to the edge losses.

A set of HAGIS simulations was carried out in which the fishbone amplitude parameter $A_{\text {sat }}\left(\equiv \delta \tilde{B}_{r, \max } / B_{0}\right)$ was scanned (see also figure 8 for $A_{\text {sat }}$ definition). A comparison of measured and predicted proton losses per fishbone as a function of the fishbone amplitude is shown in figure 17. For the experimental data in this plot, the magnitude of the measured losses (scaled in a.u.) is obtained by integrating the photomultiplier signals of the scintillator diagnostic (corrected for their relative PM sensitivities) over the time interval of the fishbone. Similarly, the fishbone amplitude values have been inferred from electron temperature profile displacements (measured with an array of ECE radiometers) using the expression

$$
\xi=\frac{\delta T_{e}}{\left|\nabla T_{e}\right|}
$$

(which neglects plasma compressibility [39]) and then linked to the quantity $\delta \tilde{B}_{r, \max } / B_{0}$ through Poincare plots of the perturbed magnetic field lines inside the $q=1$ rational surface. (Generally speaking, the signal of the ECE diagnostic is a mixture of fluctuations of temperature, density and magnetic field. However, density fluctuations can be neglected because at this location the plasma is optically thick. The distortion of the total magnetic field (ECE measurement position) as a result of the perturbation is negligible as well, because the perturbation $\left(\delta B_{r}\right.$ and $\delta B_{\text {pol }}$ are of order $\left.10^{-2} B\right)$ is 
perpendicular to the main component of $B$. This implies that $B=\left[B_{\text {tor }}^{2}+\left(B_{\text {pol }}+\right.\right.$ $\left.\left.\delta B_{\mathrm{pol}}\right)^{2}+\delta B_{r}^{2}\right]^{1 / 2}$ changes only by order $10^{-4}$.) This was done for a sample of 26 fishbones in discharge $69100(t=22.170-23.110 \mathrm{~s})$. The obtained amplitudes $\delta \tilde{B}_{r, \max } / B_{0}$ range from $0.3-1.2 \cdot 10^{-2}$. A polynomial fit to the experimental data with linear and quadratic components yields essentially a purely quadratic increase of the losses with amplitude over the entire amplitude range. For the simulation data, a quadratic dependence of the losses is found as well at amplitudes $\delta \tilde{B}_{r, \max } / B_{0}>0.75 \cdot 10^{-2}$. However, for $\delta \tilde{B}_{r, \max } / B_{0}<0.75 \cdot 10^{-2}$ the simulations predict a linear dependence (highlighted by the red line) which is not corroborated by the experiment. (The change from linear to quadratic losses with increasing mode amplitude can be understood in terms of a transition from near-boundary losses to stochastic (diffusive) losses, as described in [40]). So far the reason for this difference could not be identified. One option would be that by ignoring plasma compressibility the fishbone amplitudes are being underestimated. The fishbone frequency is far too low for perpendicular compressibility to play a role, but parallel compressibility may not be negligible. This is because the fishbone frequency is comparable to the sound frequency, especially towards the end of the fishbone cycle. Nevertheless, in order to be consistent with the simulations the experimental fishbone amplitudes would need to be larger by about a factor 2, which is considerable. For completeness it is finally noted that, although in the plot only amplitudes up to $1.50 \cdot 10^{-2}$ are shown, for the simulated data the amplitude was varied over a wider range (up to $\left.\delta \tilde{B}_{r, \max } / B_{0}=3 \cdot 10^{-2}\right)$. Above $\sim 2.0 \cdot 10^{-2}$, the predicted losses deviate from the scaling shown due to the onset of magnetic field line stochasticity (leading to additional losses), but none of the fishbones in discharge 69100 is found to reach this regime.

\section{Summary and Conclusions}

In this work we have reported on the occurrence of enhanced fast ion losses in the presence of beam-driven fishbone oscillations on JET, whose energy $(\sim 0.5-4 \mathrm{MeV})$ is found to be approximately one order of magnitude higher than the energy of the injected beam ions (max. $130 \mathrm{keV}$ ) driving the fishbone. In the particular discharge scrutinised here, the measured losses are unambiguously identified as trapped fast protons originating from RF hydrogen minority heating. This phenomenon is of interest per se because of its possible impact on future burning plasma devices, and has been characterised in detail in the first part of this article.

In the second part of this article, results from realistic numerical simulations were presented. The computations were performed through a combined use of the HAGIS, SELFO (validated against NPA measurements) and MISHKA codes. Two classes of losses are identified: (a) from trapped protons near the plasma boundary which become lost due to stochastic orbit diffusion, and (b) from counter-passing protons deep in the plasma core which due to orbit stochasticity transit into an unconfined trapped orbit. For the range of fishbone amplitudes observed in this discharge magnetic field line stochasticity is not an issue. It is worth noting that the orbit loss mechanisms found 
here closely resemble earlier findings on the effect of (Kadomtsev-type) sawtooth crashes on fusion product losses in TFTR [41]. This is not surprising, given that the underlying mode is the internal kink mode in both cases. Our simulations have further shown that (apart from rare exceptions) the losses are non-resonant, i.e. the orbit motion does not resonate with the wave field for any of the frequencies covered by the fishbone frequency chirp.

The following quantities are accessible from the experiment for a particular location inside the torus and have been compared with the numerical predictions: energy distribution of the losses, pitch angle distribution of the losses, temporal behaviour of the losses during a fishbone cycle, and scaling of the losses with the fishbone amplitude. For this comparison a number of approximations had to be made to overcome either code or computational time limitations (even with these approximations, the simulations are still computationally expensive). For convenience, the approximations used in our model are recapitulated here:

- the linear MHD eigenfunction of the internal mode is used, non-linear or kinetic modifications to the eigenmode structure are not considered.

- frequency and amplitude evolution of the fishbone are specified analytically ("typical" fishbone)

- there is no continuous replenishment of the fast particle distribution by ICRH

- plasma compressibility is ignored

- as ion orbits could not be traced outside the separatrix all the way up to the probe, it was stipulated that in order to be considered, ions had to cross the separatrix in the poloidal vicinity of the probe; toroidal symmetry is assumed.

Using these approximations, we find that many of the simulation predictions are either in broad agreement (time distribution of losses, dependence on fishbone amplitude, pitch angle distribution of losses) or even in very good agreement (energy distribution of losses) with the experiment, but also some features have been found that could not be reconciled with the experiment (time of losses peak in fishbone cycle, predicted linear scaling of losses with fishbone amplitude for the low range of fishbone amplitudes). For the pitch angle distribution of losses a satisfactory agreement (at best) is only obtained when losses from counter-passing ions in the plasma core are ignored. We note that this is found to be the case not only for the fishbone amplitude used for figure 16, but also for simulations with other fishbone amplitudes. At this point in time it is unclear whether the unexplained features are due to deficiencies in our model, or because the simulation codes do not yet incorporate all the relevant physics. This comparison exercise should be considered work in progress. Further tests with alternative (refined) models are anticipated.

[19] [20].

One of the goals of this work was to devise a numerically tractable but at the same time sufficiently accurate model that allows a detailed comparison with the scintillator measurements. Although some work remains to be done for the correct reproduction of 
the results with our modelling tools, good progress has been made. One of the next steps will be the application of this technique to the investigation of more complex phenomena, such as Alfven Eigenmodes or Alfven Cascades. Dedicated JET experiments have been recently performed to study the influence of these instabilities on the fast ion confinement deterioration and loss [42], which will be the subject of a future publication.

\section{Acknowledgments}

This work, supported by the European Communities under the contract of Association between EURATOM and Max-Planck Institut für Plasmaphysik, was carried out within the framework of the European Fusion Development Agreement. The views and opinions expressed herein do not necessarily reflect those of the European Commission.

\section{References}

[1] R. B. White et al. Phys. Plasmas, 2:2871, 1995.

[2] W. W. Heidbrink and G. J. Sadler. Nucl. Fusion, 34:535, 1994.

[3] A. Fasoli et al. Progress in the iter physics basis chapter 5: Physics of energetic ions. Nucl. Fusion, 47:S264, 2007.

[4] K. McGuire et al. Phys. Rev. Lett., 50:891, 1983.

[5] L. Chen, R. B. White, and M. N. Rosenbluth. Phys. Rev. Lett., 52:1122, 1984.

[6] R. B. White, R. J. Goldston, K. McGuire, A. H. Boozer, D. A. Monticello, and W. Park. Phys. Fluids, 26:2958, 1983.

[7] R. B. White and M. S. Chance. Phys. Fluids, 27:2455, 1984.

[8] B. Coppi and F. Porcelli. Fusion Technology, 13:447, 1988.

[9] W. W. Heidbrink, R. Hay, and J. D. Strachan. Phys. Rev. Lett., 53:1905, 1984.

[10] H. H. Duong and W. W. Heidbrink. Nucl. Fusion, 33:211, 1993.

[11] S. J. Zweben et al. Nucl. Fusion, 30:1551, 1990.

[12] S. Baeumel et al. Rev. Scient. Instr., 75:3563, 2004.

[13] S. J. Zweben. Nucl. Fusion, 29:825, 1989.

[14] M. García-Muñoz et al. Nucl. Fusion, 47:L10, 2007.

[15] M. Isobe et al. Rev. Scient. Instr., 70:827, 1999.

[16] M. Nishiura, M. Isobe, T. Saida, M. Sasao, and D. S. Darrow. Rev. Scient. Instr., 75:3646, 2004.

[17] A. Werner, A. Weller, D. S. Darrow, and the W7-AS Team. Rev. Scient. Instr., 72:780, 2001.

[18] M. J. Berger, J. S. Coursey, M. A. Zucker, and J. Chang. ESTAR, PSTAR, and ASTAR: Computer Programs for Calculating Stopping-Power and Range Tables for Electrons, Protons, and Helium Ions (version 1.2.3). [Online] Available: http://physics.nist.gov/Star [2009, April 24]. National Institute of Standards and Technology, Gaithersburg, MD., 2005.

[19] D. Darrow et al. Fus. Eng. and Design, 74:853, 2005.

[20] G. Bonheure et al. Proc. 36th EPS Conf. on Plasma Physics, Sofia, 2009 ECA, Vol., 33A:P2.145, 2009.

[21] A. B. Izvozchikov et al. JET Report JET-R(91)12, 1991.

[22] V. I. Afanasyev et al. Rev. Scient. Instr., 74:2338, 2003.

[23] K. G. McClements, R. O. Dendy, and A. Gondhalekar. Nucl. Fusion, 37:473, 1997.

[24] J. Hedin, T. Hellsten, L.-G. Eriksson, and T. Johnson. Nuclear Fusion, 42:527, 2002.

[25] L.-G. Eriksson, T. Hellsten, and U. Willén. Nucl. Fusion, 33:1037, 1993.

[26] R. J. Goldston, D. C. McCune, H. H. Towner, S. L. Davis, R. J. Hawryluk, and G. L. Schmidt. J. Comp. Physics, 43:61, 1981. 
[27] D. Borba et al. Nucl. Fusion, 40:775, 2000.

[28] D. P. O’Brien, L. L. Lao, E. R. Solano, M. Garribba, T. S. Taylor, J. G. Cordey, and J. J. Ellis. Nucl. Fusion, 32:1351, 1992.

[29] M. Brix, N. C. Hawkes, A. Boboc, V. Drozdov, S. E. Sharapov, and JET EFDA contributors. Rev. Scient. Instr., 79:10F325, 2008.

[30] M. Brix, A. Boboc, and JET-EFDA contributors. Calculation of electron density profiles from line integrated interferometer measurements at jet. to be submitted to Plasma Phys. Control. Fusion, 2010.

[31] A. B. Mikhailovskii et al. Plasma Phys. Rep., 23:844, 1997.

[32] A. Ödblom, B. N. Breizman, S. E. Sharapov, T. C. Hender, and V. P. Pastukhov. Phys. Plasmas, 9:155, 2002.

[33] S. D. Pinches et al. Comp. Phys. Comm., 111:133, 1998.

[34] T. Hellsten, J. Carlsson, and L.-G. Eriksson. Phys. Rev. Lett., 74:3612, 1995.

[35] L. Villard, K. Appert, R. Gruber, and J. Vaclavik. Computer Physics Reports, 4:95, 1986.

[36] L. Villard, S. Brunner, and J. Vaclavik. Nuclear Fusion, 35:1173, 1995.

[37] J. A. Heikkinen and S. K. Sipilä. Phys. Plasmas, 2:3724, 1995.

[38] V. A. Yavorskij, D. Darrow, V. Ya. Goloborod'ko, S. N. Reznik, U. Holzmueller-Steinacker, N. Gorelenkov, and K. Schoepf. Nucl. Fusion, 42:1210, 2002.

[39] C. Z. Cheng. Phys. Rep., 211:1, 1992.

[40] C. T. Hsu and D. J. Sigmar. Phys. Fluids B, 4:1492, 1992.

[41] Ya. I. Kolesnichenko, V. V. Lutsenko, R. B. White, Yu. V. Yakovenko, and S. J. Zweben. Phys. Plasmas, 6:1117, 1999.

[42] V. G. Kiptily et al. Nucl. Fusion, 49:065030, 2009. 